\title{
EDITORIAL
}

\section{EL PROGRAMA DE ACCIÓN DE LA UNIÓN EUROPEA EN EL ÁMBITO DE LA SALUD PÚBLICA}

\author{
Antonio Calvete \\ Unidad de Coordinación de la Unión Europea. Subdirección General de Promoción de la Salud y Epidemiología. \\ Dirección General de Salud Pública. Ministerio de Sanidad y Consumo.
}

Las autoridades sanitarias tienen, entre otras responsabilidades, las de garantizar en la medida de lo posible que los productos y servicios comercializados cumplan con unos requisitos de seguridad elevados; que los ciudadanos gocen de un ambiente sano; que los lugares de trabajo sean seguros e higiénicos; que la asistencia sanitaria sea fiable y de alta calidad, etcétera. También la Unión Europea tiene, en el ámbito de la salud pública, las obligaciones que los Tratados le asignan. Así, el artículo 3 p) del Tratado Constitutivo de la Comunidad Europea determina que la Comunidad, para cumplir sus fines, contribuirá al logro de un alto nivel de protección de la salud. Y a la hora de concretar ese propósito en el artículo 152, establece que:

1. al definirse y ejecutarse todas las políticas y acciones de la Comunidad se garantizará un alto nivel de protección de la salud humana;

2. la acción de la Comunidad, que complementará las políticas nacionales, se encaminará a mejorar la salud pública, prevenir las enfermedades humanas y evitar las fuentes de peligro para la salud humana;

3. los Estados miembros, en colaboración con la Comisión, coordinarán entre sí sus políticas y programas res- pectivos en los ámbitos a que se refiere el apartado anterior;

4. el Consejo contribuirá a la consecución de los objetivos del presente artículo adoptando:

- medidas que establezcan altos niveles de calidad y seguridad de los órganos y sustancias de origen humano, así como la sangre y derivados de la sangre;

- medidas en los ámbitos veterinario y fitosanitario que tengan como objetivo directo la protección de la salud pública;

- medidas de fomento destinadas a proteger y mejorar la salud humana, con exclusión de cualquier armonización de las disposiciones legales y administrativas de los Estados miembros.

5. La acción comunitaria en el ámbito de la salud pública respetará plenamente las responsabilidades de los Estados miembros en materia de organización y suministro de servicios sanitarios y asistencia médica.

De la lectura de lo anterior se desprende que la Comunidad Europea tiene una serie de posibilidades a la hora de actuar en el ámbito de la salud pública y también una serie de 
limitaciones. En cuanto a las posibilidades cabe señalar que se podrá elaborar legislación comunitaria, de obligado cumplimiento por tanto para los Estados miembros, en cuanto a calidad y seguridad de los órganos y sustancias de origen humano y en los ámbitos veterinario y fitosanitario; prueba de ello es la Directiva, recientemente aprobada, por la que se establecen normas de calidad y de seguridad para la extracción, verificación, tratamiento, almacenamiento y distribución de sangre humana y sus componentes, y la propuesta de Directiva, actualmente en proceso de debate en el Consejo y en el Parlamento Europeo, relativa al establecimiento de normas de calidad y de seguridad para la donación, la obtención, la verificación, el procesamiento, el almacenamiento y la distribución de células y tejidos humanos.

En cuanto a las limitaciones, es necesario tener en cuenta que para la Comunidad, fuera de los ámbitos señalados en el citado artículo 152, sólo será posible adoptar «medidas de fomento», es decir, recomendaciones sin fuerza legal para obligar a los Estados miembros o disposiciones basadas en otros artículos del Tratado y, por tanto, no dirigidas a proteger y mejorar la salud humana, aunque influyan positivamente en el logro de ese objetivo. Esto es importante a la hora de responder a determinadas críticas que achacan a la Comunidad Europea falta de interés para llegar más lejos en determinados aspectos que tienen que ver con la salud; no se puede olvidar que, en última instancia, lo establecido en los Tratados comunitarios es la expresión de las competencias que los Estados miembros han querido ceder a la Comunidad o han querido reservarse para sí.

En este contexto, conviene recordar que el Programa se aprueba mediante una Decisión, instrumento legal comunitario que es de obligado cumplimiento para los Estados miembros.

El 24 de noviembre de 1993 la Comisión presentó una Comunicación en la que propo- nía un Plan de salud pública que comprendía ocho programas sectoriales:

- En el año 1996 se aprobaron los de

- Promoción, información, educación y formación en materia de salud;

- Lucha contra el cáncer;

- Prevención del sida y otras enfermedades transmisibles.

- En 1997

- Prevención de la toxicomanía;

- Vigilancia de la salud.

- Y en 1999

- Prevención de lesiones;

- Enfermedades poco comunes;

- Enfermedades relacionadas con la contaminación.

El 17 de junio de 1997 se firmó el Tratado de Amsterdam, que modificó el de Maastricht, pasando a regirse la salud pública comunitaria por el artículo 152 que ya hemos comentado más arriba y que ampliaba las competencias comunitarias. Este hecho, unido a los nuevos desafíos para la salud pública y a la experiencia acumulada, hicieron que la Comisión elaborara otra Comunicación en 1998 en la que indicaba la necesidad de una nueva estrategia y un nuevo programa en el ámbito de la salud pública, con un planteamiento integral que sustituyera a los ocho programas sectoriales vigentes. El Consejo aceptó el planteamiento y animó a la Comisión a trabajar en esa línea.

Como consecuencia, la Comisión publicó el 16 de mayo de 2000 una Comunicación sobre la estrategia sanitaria de la Comunidad Europea acompañada, como expresión de dicha estrategia, por una propuesta de Decisión para la adopción de un programa de acción comunitario en el ámbito de la salud 
pública. La nueva estrategia comunitaria en salud pública incluye dos elementos: en primer lugar, un marco de salud pública compuesto por el programa de acción y por la política y legislación correspondientes en el ámbito de la salud pública; en segundo lugar, la contribución de las restantes políticas y actividades comunitarias para conseguir un nivel elevado de protección de la salud de manera integral e integrada.

\section{El programa comunitario de acción en el ámbito de la salud pública (2001-2006)}

El programa lo aprobó de hecho el Consejo de Ministros de Salud de la UE en su sesión número 2440 de 26 de junio de 2002 bajo presidencia española, aunque de derecho se adopta, una vez que lo aprueba también el Parlamento Europeo, por medio de la Decisión 1786/2002/CE del Parlamento Europeo y del Consejo de 23 de septiembre de 2002, la cual se publicó en el Diario Oficial de las Comunidades Europeas y, por tanto, entró en vigor el 9 de octubre de 2002.

Los objetivos generales o líneas maestras del programa son:

1. Mejorar la información y los conocimientos sanitarios para fomentar la salud pública: La intención es desarrollar un sistema de vigilancia de la salud sostenible y un sistema de información para la detección y el seguimiento de todo tipo de riesgos sanitarios.

2. Aumentar la capacidad de afrontar de manera rápida y coordinada las amenazas para la salud: Mediante el desarrollo, refuerzo y apoyo de la capaci$\mathrm{dad}$, el funcionamiento y la interconexión de los mecanismos de alerta precoz y respuesta rápida. Esto se hará, principalmente, aunque no sólo, apoyando a la red comunitaria de vigilancia epidemiológica y de control de las enfermedades transmisibles creada por la Decisión 2119/98/CE.
3. Abordar los factores determinantes de la salud: Se fomentará el desarrollo de actividades de promoción de la salud y actividades preventivas de amplio alcance, así como la utilización de instrumentos específicos de reducción y eliminación de riesgos.

En el cumplimiento de esos tres objetivos, el programa contribuirá a:

- Asegurar un nivel elevado de protección de la salud humana en la definición y ejecución de todas las políticas y acciones de la Comunidad, mediante el fomento de una estrategia de la salud integrada e intersectorial;

- Reducir las desigualdades en materia de salud;

- Fomentar la cooperación entre los Estados miembros en los ámbitos regulados por el artículo 152 del Tratado.

El programa también se plantea, en cumplimiento del primer punto del Artículo 152 del Tratado («al definirse y ejecutarse todas las políticas y acciones de la Comunidad se garantizará un alto nivel de protección de la salud humana»), la realización de estrategias $\mathrm{y}$ acciones conjuntas con otros programas $\mathrm{u}$ organismos comunitarios relacionados o con incidencia en la salud: protección de los consumidores, protección social, seguridad y salud laboral, investigación y desarrollo tecnológico, medio ambiente...

La Comisión Europea es la responsable de la aplicación del programa y lo hace por medio de un Comité presidido por ella y formado por representantes de los Estados miembros. Entre las funciones de este Comité está la elaboración de un plan de trabajo anual que ha de determinar las prioridades en cuanto a las acciones que se han de llevar a cabo, incluido la asignación de fondos a cada una de ellas. También se ocupará este Comité de aplicar las estrategias y acciones 
conjuntas de las que se hablaba en el párrafo anterior, de tomar las medidas necesarias para evaluar el programa y de establecer los métodos de transmisión, intercambio y comunicación de información y de reacción rápida ante riesgos sanitarios.

Las áreas prioritarias se determinan en el plan de trabajo anual del Comité. Para el año 2003 se han establecido las siguientes:

1. Información sanitaria. Comprende acciones en los siguientes ámbitos:

- Desarrollo y coordinación del sistema de información sanitaria;

- Funcionamiento del sistema de vigilancia de la salud;

- Puesta a punto de mecanismos para la realización de informes y análisis sobre las cuestiones sanitarias y la elaboración de informes de salud pública;

- Mejora del acceso a los datos y transferencia de los mismos a escala de la UE: el portal de la salud pública en la UE y otras plataformas de publicación.

2. Amenazas para la salud. Comprende acciones en los siguientes ámbitos:

- Vigilancia (proseguir la puesta en práctica de la Red de vigilancia epidemiológica y de control de las enfermedades transmisibles);

- Alerta y reacción rápidas;

- Actividades relacionadas con la seguridad sanitaria y la preparación para afrontar posibles amenazas por agentes químicos o biológicos;

- Seguridad de la sangre, los tejidos y los órganos;

- Resistencia a los antimicrobianos;

- Apoyo al trabajo en red de los laboratorios;
- Desarrollo de capacidades (reforzar la formación en materia de amenazas para la salud);

- Enfermedades poco comunes.

3. Factores determinantes de la salud. Comprende acciones en los siguientes ámbitos:

- Nutrición y actividad física;

- Tabaco;

- Alcohol;

- Drogas;

- Salud mental;

- Salud sexual y reproductiva;

- Medio ambiente;

- Promoción de la salud en contextos específicos y en el lugar de trabajo;

- Formación en salud pública;

- Acción sobre las lesiones y la reducción del riesgo de lesiones.

4. Temas transversales. Comprende acciones en los siguientes ámbitos:

- Evaluación de los efectos sanitarios;

- La salud en los países candidatos;

- Reducir las desigualdades en materia de salud;

- Cooperación entre los Estados miembros en materia de servicios sanitarios;

- Promoción de las mejores prácticas y de la eficacia;

- El envejecimiento y la salud.

El presupuesto inicialmente previsto por la Comisión fue de 300 millones de euros, pero algunas delegaciones pretendían que no pasara de 280, mientras que otras llegaban hasta los 400. Finalmente, después de arduas discusiones, quedó fijado en 312 millones para los seis años de duración prevista. 
La Decisión que crea el programa prevé la forma en que se hará la evaluación y la difusión de los resultados del mismo. La Comisión, que es la responsable de ello con la colaboración de los Estados miembros que han de facilitar la información necesaria para hacerlo, informará cada año al Comité. También, al final del cuarto año de desarrollo del programa y a finales del año siguiente al de su finalización, la Comisión entregará al Parlamento Europeo, al Consejo, al Comité Económico y Social y al Comité de las Regiones, sendos informes realizados por expertos independientes con la evaluación de la ejecución y de los logros de cada período.

El programa estará en vigor desde el 1 de enero de 2003 hasta el 31 de diciembre de 2008.

A la hora de concluir, parece necesario volver sobre las ideas que se apuntaban en la introducción respecto a las posibilidades y limitaciones de la Comunidad en cuanto a su actuación en relación con la salud pública. Podríamos decir lo mismo en cuanto al programa del que estamos hablando, dado que muchos pueden pensar que no ha ido todo lo lejos que hubiera sido de desear. Pero también, en mi opinión, podemos decir que ha llegado a lo más que le permite la base jurídica en la que se apoya.

Con el Programa comunitario en la mano, las instituciones y los profesionales interesados de los Estados miembros podrán presentar proyectos y solicitar financiación respecto a un amplio número de ámbitos, como los que se han relacionado más arriba.
También parece importante destacar la continuidad de los trabajos en marcha, basados en los ocho antiguos programas de salud (cáncer, sida, promoción de salud, etc.) que son derogados al iniciarse éste. Esto se contempla en el párrafo 6 del artículo 5 de la Decisión 1786/2002/CE que dice: La Comisión, en estrecha cooperación con los Estados miembros, asegurará la transición entre las acciones que se desarrollen en el marco de los programas derogados en el artículo 13 de esta misma Decisión, y las que se apliquen en el contexto del presente programa. Esto significa que los proyectos realizados como desarrollo de alguno de esos programas podrán continuar en la medida en que encajen en las acciones previstas en el actual.

En definitiva, contamos a partir de ahora con una herramienta que, potencialmente, tiene una enorme utilidad para el desarrollo de la salud pública en cada uno de los Estados miembros de la Unión Europea. Depende ahora de todos los implicados en ese desarrollo -autoridades, instituciones públicas y privadas y profesionales- sacarle el máximo rendimiento para que los resultados sean positivos para los destinatarios, esto es, para todos los ciudadanos europeos.

\section{BIBLIOGRAFÍA}

1. Tratado constitutivo de la Comunidad Europea (versión consolidada). Diario Oficial de las Comunidades Europeas C 325 de 24 de diciembre de 2002.

2. Decisión n ${ }^{\circ}$ 1786/2002/CE del Parlamento Europeo y del Consejo de 23 de septiembre de 2002 relativa a la adopción de un programa de acción comunitario en el ámbito de la salud pública. Diario Oficial de las Comunidades Europeas L 271 de 9 de octubre de 2002. 